\title{
Can eczema be a marker for interal malignancy?
}

\author{
Authors: Ana Gašić ${ }^{1}$, Ivana Karla Franić ${ }^{1}$, Ana Giljanović ${ }^{1}$, Liborija Lugović-Mihić ${ }^{2}$ (mentor) \\ ${ }^{1}$ School of Medicine, University of Zagreb, Zagreb, Croatia \\ ${ }^{2}$ Clinical Department of Dermatovenereology, Sestre Milosrdnice University Hospital Centre, Zagreb, \\ Croatia
}

DOI: https://doi.org/10.26800/LV-142-supp5-1

\section{Background:}

Paraneoplastic disorders are a group of uncommon symptoms assumed to occur due to the substances released by the tumour or due to abnormal immune system response in patients with internal malignancy. Some neoplastic diseases may present with various cutaneous manifestations. Although these dermatoses are relatively rare, their identification can contribute to earlier diagnosis and a better outcome.

\section{Case presentation:}

A 57-year-old male patient presented with recurrent eczematous lesions on the hands and face with a concomitant itch, which has lasted several years. Although he was treated with systemic and topical corticosteroids with antihistamines numerous times, skin manifestations relapsed after drug discontinuation. Skin biopsy indicated psoriasiform dermatitis, and allergy tests were negative except positive patch test to contact allergens. The introduction of immunosuppressants was considered. However, his dermatologist insisted on extended diagnostics to screen for an eventual underlying malignancy and found slightly elevated CEA (carcinoembryonic antigen), LDH (lactate dehydrogenase), and CYFRA 21-1 (cytokeratin fragment). Due to the persistence of the lesions, other specialists were additionally consulted (otorhinolaryngologist, haematologist, urologist, and gastroenterologist who explained increased markers by his smoking habit), but they considered there was no need for further diagnostics/examinations. However, the dermatologist insisted on a PET-CT scan, and its result was normal. Finally, he insisted on esophagogastroduodenoscopy with biopsy, which verified gastric adenocarcinoma. After completing oncologic therapy both diseases went into remission.

\section{Conclusion:}

The patient suffered from unrecognized cutaneous paraneoplastic eczema, which was a consequence of gastric adenocarcinoma. Oncologic treatment was performed, and he recovered well. So, in the treatment of various skin manifestations, the possibility of internal malignancy should be considered. However, currently, there are no specific guidelines on when and which diagnostic procedures should be performed.

Keywords: immunosuppressants, internal malignancy, paraneoplastic disorder 